\title{
MIMO Device Performance Testing with the Wireless Cable Method
}

\author{
Wei Fan ${ }^{1}$, Pekka Kyösti ${ }^{2,3}$, Lassi Hentilä ${ }^{2}$, Fengchun Zhang ${ }^{1}$, and Gert F. Pedersen ${ }^{1}$ \\ ${ }^{1}$ Department of Electronic Systems, Aalborg University, Denmark \\ Email: wfa@es.aau.dk \\ ${ }^{2}$ Keysight Technologies Finland Oy, Oulu, Finland, \\ ${ }^{3}$ Centre for Wireless Communications, University of Oulu, 90014 Oulu, Finland
}

\begin{abstract}
Conducted cable testing has been dominantly utilized in the industry to evaluate multiple-input multiple-output (MIMO) capable mobile terminals. However, direct RF cable connection introduces many practical problems. Therefore, radiated testing, where built-in antennas on the mobile terminal is utilized as RF interface to receive desired testing signals, is highly desirable. Wireless cable method is a radiated testing method, where signals can be guided over-the-air to respective antenna ports, just like in the conducted cable case but without RF cable connection. In this paper, several different ways to achieve wireless cable connection for mobile terminal performance testing in the radiated setup is discussed. Validation of the wireless cable method is done both in simulation and active throughput measurements. Future directions and applicability of the wireless cable method are also discussed.
\end{abstract}

\section{INTRODUCTION}

Performance testing of mobile terminal is an essential step before product approval for the final roll-out. In the current mobile terminals, each built-in antenna is equipped with a temporary antenna connector, which can be accessed via radio frequency (RF) cables for testing purposes. In such case, internal built-in antennas are bypassed, and testing signals can be guided to the respective antenna ports via RF cables directly. Conductive cable method has been dominantly utilized in industry so far for device testing [1]-[3]. Conductive testing has been seen as an attractive and acceptable choice in the early stage of chipset and baseband development. However, it necessitates breaking the mobile terminal case to have access to antenna connectors and presents many other shortcomings.

Over-the-air (OTA) radiated testing, which eliminates the need for RF cable connections, is getting important [3]-[5]. For OTA testing of MIMO terminals, various methods were proposed, which differs in how spatial propagation channels can be emulated. Further, among the mentioned, only the multi-probe anechoic chamber (MPAC) and radiated-two stage (also called wireless cable) methods are shown to be capable of emulating arbitrary spatial channel models even in principle [4]-[7]. The MPAC method enables true end-to-end performance testing of mobile terminals, since its basic idea is to reproduce real-world propagation environments in the anechoic chamber (i.e. virtual drive testing).

Similar to cable testing, the objective of the wireless cable method is to direct testing signals to the respective antenna port. However, unlike cable testing with RF cable connection, wireless cable method does not require actual cable connection, via calibrating out the transfer matrix between the OTA antenna ports and DUT antenna ports to achieve wireless cable transmission. The wireless cable concept is highly attractive. With this concept, the number of required $\mathrm{RF}$ interface channels for the channel emulator is required only to match the number of receive $(\mathrm{Rx})$ antennas on the DUT in principle, regardless of the complexity of the chosen spatial channel model and DUT size.

In this paper, we firstly discuss different possibilities to achieve wireless cable connection for MIMO performance testing in Section II. After that, we demonstrate the effectiveness of the wireless cable methods via simulation results and active throughput measurements. In Section IV, we discuss the future work and directions of the wireless cable method.

\section{Wireless CABLE METHOD}

\section{A. Introduction}

The MIMO signal model, neglecting the noise vector at the receiver antenna port, can be written as:

$$
\boldsymbol{y}(f, t)=\boldsymbol{H}(f, t) \boldsymbol{x}(f, t),
$$

where $\boldsymbol{H}(f, t)$ denotes the time-variant radio channel transfer function between the $M$ transmit antenna ports and $N$ receive antenna ports. $\boldsymbol{H}(f, t)$ might include the effect of base station (BS) antenna characteristics (if known), the propagation channel, and the mobile station (MS) antenna characteristics (if known). $\boldsymbol{y}(f, t) \in \mathbb{C}^{N \times 1}$ and $\boldsymbol{x}(f, t) \in \mathbb{C}^{M \times 1}$ denote receive signal vector at the $N$ receive antenna ports and transmit signal vector at the $M$ transmit antenna port, respectively. We limit our discussions on $2 \times 2$ MIMO in the paper (i.e. $M=N=2$ ).

1) Conducted cable testing: Conducted testing is typically utilized to evaluate mobile terminal performance, as shown in Fig. 1. The system consists of a BS emulator, a radio channel emulator (CE) and a DUT. The BS emulator mimics the cellular network end of the link. The radio CE physically implement the mathematical representation of the channel model between the BS and the MS antenna ports $\boldsymbol{H}(f, t)$. The RF cables direct signals to the DUT antenna ports, with DUT antennas bypassed. We have 


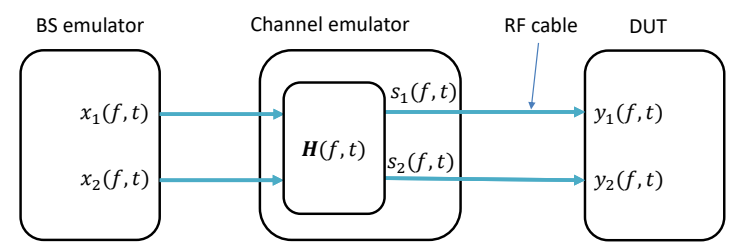

Fig. 1. System diagram of cable testing. $s_{1}(f, t)$ and $s_{2}(f, t)$ denote the desired testing signals guided/targeted to the DUT antenna port 1 and port 2 respectively.

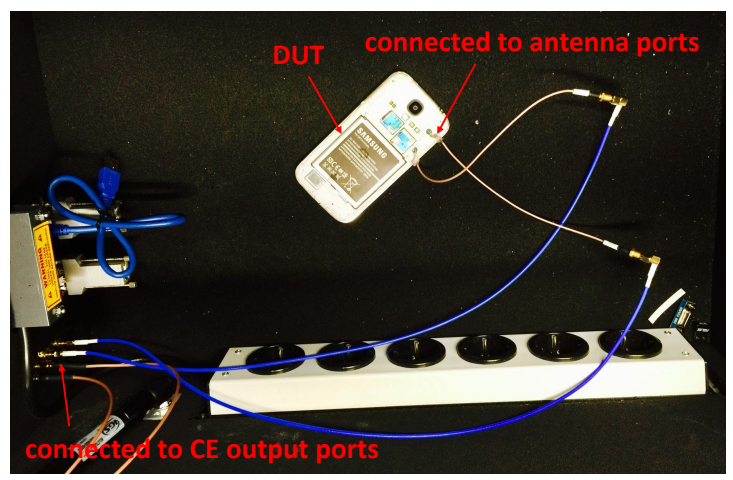

Fig. 2. An illustration of the conducted cable testing, where the handset is placed in a shielded RF enclosure.

$$
\boldsymbol{y}(f, t)=\boldsymbol{A} \boldsymbol{H}(f, t) \boldsymbol{x}(f, t)
$$

where $\boldsymbol{A} \in \mathbb{C}^{2 \times 2}$ is the transfer matrix between the $\mathrm{CE}$ output ports and the DUT antenna ports, with $a_{i, j}$ the complex transfer coefficient between the $j$-th CE output port and the $i$-th DUT antenna port, $i, j \in[1,2]$. The cross-talk between $\mathrm{RF}$ cables can be ignored and therefore we typically have

$$
\boldsymbol{A}=\left[\begin{array}{cc}
a_{11} & 0 \\
0 & a_{22}
\end{array}\right]
$$

The cable response can be easily measured and compensated in the CE. The cable setup is simple, since we can easily have desired signals at the DUT antenna port undistorted. The cost is low, since only a few RF cables and respective CE resource are needed when the antenna count on the DUT is low. As discussed in [8]-[10], there are, however, many problems with conducted the cable setup.

2) Antenna Coupler: As discussed in the cable testing, the objective is to guide the desired testing signals to the respective antenna ports on the DUT. This can be achieved with the help of an antenna coupler. The basic idea is that antenna couplers can be utilized to couple the testing signal to desired built-in antenna. In the ideal scenario, the coupling loss between the antenna coupler and the target built-in antenna is low and the isolation between the antenna coupler and undesired built-in antennas (i.e. cross-talk) is high.

The antenna coupler solution has been used for single antenna mobile terminals to avoid complicated wiring and handling in the conducted cable testing. Typically a moving

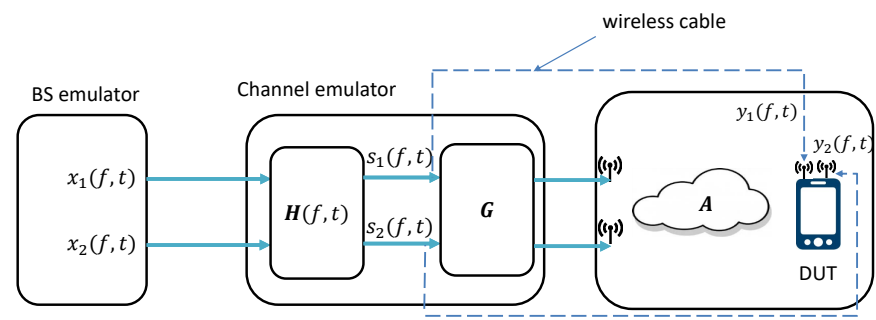

Fig. 3. System diagram of wireless cable achieved with compensating the transfer matrix between the OTA antenna ports and the DUT antenna ports.

shuttle is utilized to ensure that the best coupling position can be found for the antenna coupler. However, for multi-antenna terminals, this is more complicated. We have to minimize the coupling loss between the desired links, and maximize the isolation between the undesired links. This will get more difficult when the antenna count on the mobile terminals gets larger and antenna spacing gets smaller. For example, dual polarized co-located antennas would be difficult to handle. Though it is simple in principle and there is no need to use RF cables, practical antenna coupling solutions for multi-antenna terminals are yet to implement.

\section{B. Wireless cable method}

An illustration of the wireless cable principle is shown in Fig. 3. The DUT is placed in an RF shielded enclosure. $\boldsymbol{A}$ is the transfer matrix between the CE output ports and DUT antenna ports, which depends on the RF cables, OTA antennas, propagation between OTA antennas and DUT antennas, etc. $G$ is the calibration matrix implemented in the CE. The basic idea is that the transfer matrix $\boldsymbol{A}$, if known, can be calibrated out. The signal model can be written as:

$$
\boldsymbol{y}(f, t)=\boldsymbol{A} \boldsymbol{G H}(f, t) \boldsymbol{x}(f, t)=\boldsymbol{H}(f, t) \boldsymbol{x}(f, t)
$$

When $\boldsymbol{A G}=\boldsymbol{I}_{2}$ is met, we can achieve the functionality of conducted cable testing (i.e. achieving equivalent signal models), without RF cable connection. There are several possible ways to obtain the transfer matrix $\boldsymbol{A}$, as summarized below:

- One straightforward idea is to directly estimate the transfer matrix $\boldsymbol{A}$. Via setting channel models in a bypassed mode in the CE (i.e. $\boldsymbol{H}=\boldsymbol{I}_{2}$ ), we have $\boldsymbol{y}(f, t)=$ $\boldsymbol{A} \boldsymbol{x}(f, t)$. A popular approach in channel estimation is to utilize pilot sequence, where a known signal is transmitted and the channel matrix is estimated using the combined knowledge of the transmitted and received signal. Though the LTE receiver has the knowledge of the channel matrix between the transmitter and receiver, it is not accessible. The channel matrix is only utilized to decode the transmit signals. It is not stored or recorded, and can not be reported.

- In [11], the transfer matrix $\boldsymbol{A}$ was directly measured between the OTA antenna ports and the DUT antenna ports, via access to the DUT antenna port. This is, 
however, not suitable for handset radiated testing, since it requires access to the DUT antenna ports. The RF shielded environment where the DUT is placed does not need to be anechoic, as discussed in [11]. Basically the $\boldsymbol{A}$ can be measured over a wide bandwidth and calibrated out in the CE.

- In [6], [9], [10], [12]-[15], it was proposed that the transfer matrix $\boldsymbol{A}$ can be calculated based on the knowledge of complex OTA antenna gains, the complex propagation coefficients from OTA antennas to DUT antennas, and the complex DUT antenna gains. Complex radiation patterns and placement of all OTA antennas can be measured. A non-intrusive, complex radiation-pattern measurement of DUT antenna patterns can be realized with a special chipset functionality support. The propagation between OTA antenna and DUT antenna can be calculated if an ideal line-of-sight propagation (i.e. free space) without any reflection inside the anechoic chamber can be assumed. As can be seen, this technique requires a special baseband chipset support, which is not supported by all mobile terminal vendors and not standardized. Further, the anechoic chamber should be large enough to ensure free space propagation (i.e. no reflections and no coupling between OTA antennas). It is noted that knowledge of the DUT patterns, though difficult to obtain in practice, is valuable in antenna design and performance validation.

- In [8], a wireless cable was achieved by recording the reference signal received power (RSRP) in the LTE per DUT antenna port and selecting optimal complex weights in the $\mathrm{CE}$ output ports. The proposed method can be executed in a small RF shielded box. Further, no proprietary signal is needed in LTE systems. Instead, the communication tester can be utilized in its normal operational mode such that any commercial LTE UE can report RSRP value per antenna, the wireless cable method based on reported RSRP value per antenna works for any MIMO terminals. Further, the RF shielded enclosure can be much smaller in size, compared to that in the RTS method [9]. Below, we limit our discussions to this wireless cable method.

\section{Implementation of wireless cable method}

A possible implementation of the wireless cable method is shown in Fig. 4 (with number of OTA antenna $K=4$ ), and the system model is:

$$
\boldsymbol{y}(f, t)=\boldsymbol{A} \boldsymbol{G} \boldsymbol{H}(f, t) \boldsymbol{x}(f, t)=\boldsymbol{H}(f, t) \boldsymbol{x}(f, t),
$$

where $\boldsymbol{A} \in \mathbb{C}^{2 \times 4}$ is the unknown transfer matrix from $\mathrm{CE}$ output ports to DUT antenna ports and $G \in \mathbb{C}^{4 \times 2}$ is the routing and calibration matrix within the $\mathrm{CE}$. The routing matrix $\boldsymbol{G}$ defines on how signals $s_{1}(f, t)$ and $s_{2}(f, t)$ are assigned to CE output ports. With $K=4$, the four CE output ports are divided to two groups, each associated with two ports. The first group (transmitting $c_{1} s_{1}(f, t), c_{1} w_{1} s_{1}(f, t)$ ) is utilized to realize the first wireless connection to DUT antenna 1 , while the second group (transmitting $c_{2} s_{2}(f, t), c_{2} w_{2} s_{2}(f, t)$ ) is utilized to realize the second wireless connection to DUT antenna 2 as a group. For example, we can utilize port 1 and 2 for $c_{1} s_{1}(f, t), c_{1} w_{1} s_{1}(f, t)$ respectively as a group, and port 3 and 4 for $c_{2} s_{2}(f, t), c_{2} w_{2} s_{2}(f, t)$ respectively as another group. We have

$$
\boldsymbol{G}=\left[\begin{array}{cc}
c_{1} & 0 \\
c_{1} w_{1} & 0 \\
0 & c_{2} \\
0 & c_{2} w_{2}
\end{array}\right]
$$

From hardware implementation point of view, routing matrix can be easily realized as shown in Fig. 4, where weights at CE output ports 1-4 are to be determined in the calibration procedure. The calibration procedure is detailed in [8] and only outlined here:

1) Locate the DUT in a RF shielded box.

2) Set channels in a bypassed mode in the CE (i.e. $\boldsymbol{H}=$ $\boldsymbol{I}_{2}$ ), and establish a call in the spatial multiplexing mode.

3) Find optimal complex weight $w_{1}$ to establish the first wireless cable transmission:

a) Enable CE output ports transmitting $s_{1}(f, t)$ and $\left.w_{1} s_{1}(f, t)\right)($ i.e. port 1 and 2 ), and disable $\mathrm{CE}$ output ports transmitting $s_{2}(f, t)$ and $w_{2} s_{2}(f, t)$ (i.e. port 3 and 4 ).

b) Sweep complex weight $w_{1}$ and record the RSRP values at the two DUT antenna port.

c) Select the optimal complex weight $w_{1}$ so that isolation $\frac{\operatorname{RSRP}_{1}\left(w_{1}\right)}{\operatorname{RSRP}_{2}\left(w_{1}\right)}$ is maximized, where $\operatorname{RSRP}_{1}\left(w_{1}\right)$ denotes the power for the direct link and $\operatorname{RSRP}_{2}\left(w_{1}\right)$ denotes the cross-talk link for the first wireless cable.

4) Similar to step 3, to establish the second wireless cable transmission, we can find the optimal complex weight $w_{2}$ so that isolation $\frac{\operatorname{RSRP}_{2}\left(w_{2}\right)}{\operatorname{RSRP}_{1}\left(w_{2}\right)}$ is maximized.

5) Analogous to ensure same cable loss for cables in the conductive testing, we need to determine $c_{1}$ and $c_{2}$ so that we can ensure that propagation coefficients for the desired direct links are balanced. By doing this we also compensate out possible gain imbalances of DUT RF chains. This is a downside of the method compared to the MPAC method. It is noted that the DUT antennas are not inherently included in the testing, similar to the cable testing.

6) After the calibration stage, a wireless cable connection (i.e. low cross-talk and balanced branches) is established, via setting the weights for each $\mathrm{CE}$ output port. We can perform actual throughout measurements with desired channel models $\boldsymbol{H}(t, f)$.

A photo of the practical setup for wireless cable setup is shown in Fig. 5. 


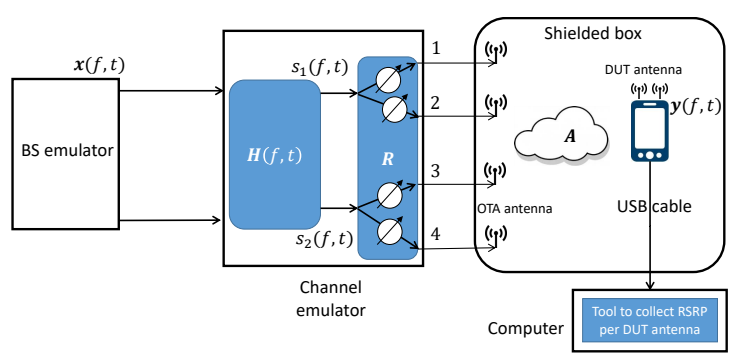

Fig. 4. System diagram of wireless cable testing implementation.

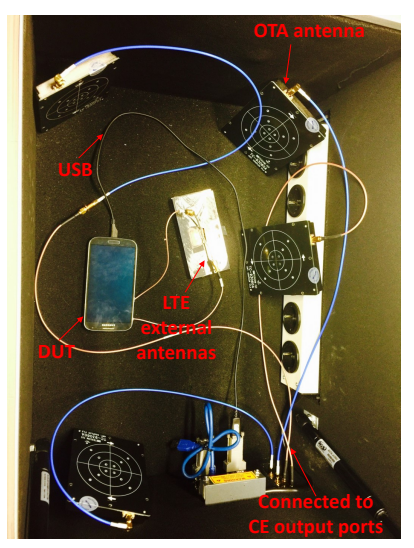

Fig. 5. A photo of the wireless cable measurement setup.

\section{Algorithm VALidAtion}

\section{A. Validation in Simulations}

In this section, we assume $M=N=K=2$, and the calibration matrix inside the CE can be implemented as $\boldsymbol{G}=$ $\left[c_{1}\left(\begin{array}{c}1 \\ w_{1}\end{array}\right) c_{2}\left(\begin{array}{c}1 \\ w_{2}\end{array}\right)\right]$. The objective is to investigate whether we can find the complex weights $w_{1}, w_{2}$, and gain weights $c_{1}, c_{2}$ to ensure $\boldsymbol{A} \boldsymbol{G}$ approximates an identity matrix (i.e. achieving wireless cable connections), independent of $\boldsymbol{A}$. As explained earlier, with the RTS method [9], the measured transfer matrix $\boldsymbol{A}$ can be directly inverted and implemented in the CE. In this work, we determine the calibration matrix $G$ based on the RSRP recorded per antenna port, without a knowledge of $\boldsymbol{A}$.

Below we intend to illustrate this by two different examples of matrix $\boldsymbol{A}$. Assume $\boldsymbol{A}=$ $\left[\begin{array}{cc}0.7485+0.3201 i & -0.0971-0.8083 i \\ 0.2515-0.3201 i & -0.0971+0.8083 i\end{array}\right]$ (with its inverse $\left.\boldsymbol{A}^{+}=\left[\begin{array}{cc}1 & 1 \\ 0.5 \cdot \exp \left(j \frac{\pi}{4}\right) & \exp \left(-j \frac{\pi}{3}\right)\end{array}\right]\right)$, following the calibration procedure [8], we can find $w_{1}=0.5 \cdot \exp \left(j \frac{\pi}{4}\right)$, $w_{2}=\exp \left(-j \frac{\pi}{3}\right)$ and $c_{1}=1, c_{2}=1$, as shown in Fig. 6. An isolation of up to $54 \mathrm{~dB}$ and $48.5 \mathrm{~dB}$ can be achieved for the two wireless cable.

$$
\text { Assume } \boldsymbol{A}=\left[\begin{array}{cc}
-0.1974+0.0620 i & 0.4571+0.4197 i \\
0.0044+0.1033 i & 0.7619+0.6996 i
\end{array}\right]
$$
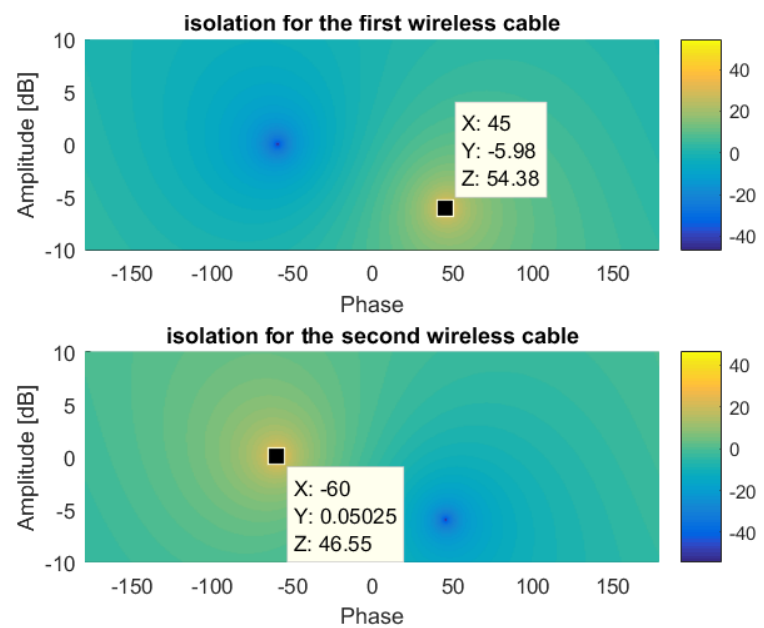

Fig. 6. Achieved isolation for the first wireless cable (top) and second wireless cable (below) with first $\boldsymbol{A}$.
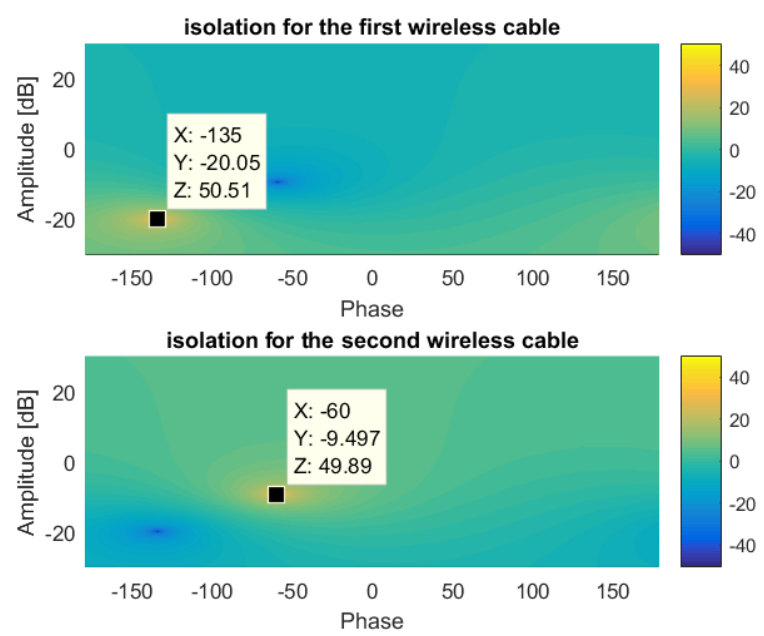

Fig. 7. Achieved isolation for the first wireless cable (top) and second wireless cable (below) with second $\boldsymbol{A}$. (with its inverse $\boldsymbol{A}^{+}=\left[\begin{array}{cc}-5 & 3 \\ 0.5 \cdot \exp \left(j \frac{\pi}{4}\right) & \exp \left(-j \frac{\pi}{3}\right)\end{array}\right]$ ),
following the calibration procedure, we can find $w_{1}=0.1$ $\exp \left(-j \frac{3 \pi}{4}\right), w_{2}=0.335 \exp \left(-j \frac{\pi}{3}\right)$ and $c_{1}=5, c_{2}=3$. As shown in Fig. 6, an isolation of up to around $50 \mathrm{~dB}$ can be achieved for the both wireless cable.

As can be seen, good wireless cable connections can be achieved for two different examples of $\boldsymbol{A}$ following the calibration procedure in the simulation. There are, however, cases where transfer function $\boldsymbol{A}$ is not favorable. For example, we can not acheive wireless cable if transfer function $\boldsymbol{A}$ is rank deficient. In these cases, e.g., rotation or displacement of the DUT could be tried. Further, we might need to sweep a large range to obtain the optimal amplitude term in the complex weight $w_{1}$ and $w_{2}$, which might be not supported due to the limited dynamic range in the CE output port. Further, it takes long calibration time to sweep a large amplitude range. 


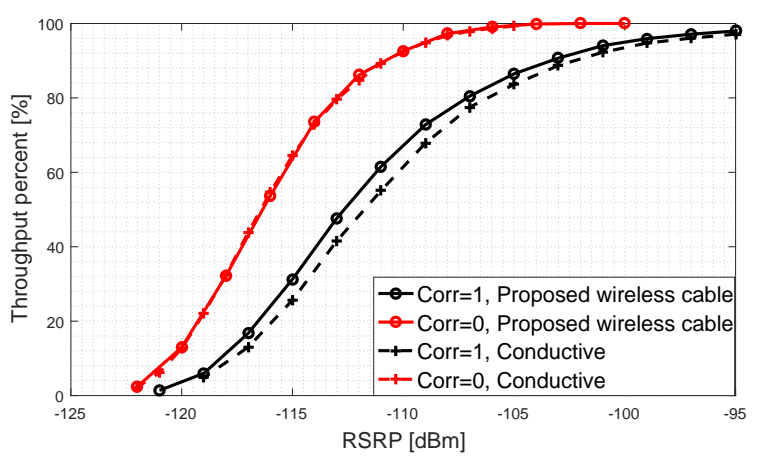

Fig. 8. Measured throughput results in uncorrelated channels and fully correlated channels in conductive and proposed setups.

\section{B. Validation in active throughput measurement}

To experimentally validate the wireless cable technique, measured throughput results in the wireless cable setup (cf. Fig 5 with $K=4$ ) were compared with results achieved in the conductive setup (cf. Fig 2).

Two representative $2 \times 2$ MIMO channel models, i.e. one uncorrelated with $\rho=0$ and the other fully correlated with $\rho=1$, are selected in the validation measurements. A BPR = $0 \mathrm{~dB}$ was set both for the conductive and wireless cable setup in the validation measurements. The validation measurement results are shown in Figure 8. The measured throughput in uncorrelated channels is higher than in the fully correlated channels with same RSRP values, as expected.

For the uncorrelated channel model, an excellent agreement is achieved for the throughput results measured in the conductive and in the proposed setup, as shown in Figure 8. A small deviation between the measured throughput results exist in the two setups in fully correlated channels. The validation measurement results demonstrated that the proposed setup can replace the conductive setup. It is noted that the achieved isolation was $I_{1}=34.3 \mathrm{~dB}$ and $I_{2}=25 \mathrm{~dB}$, respectively for the wireless cable connections in the measurement.

\section{Discussions AND FUTURE WORK}

The wireless cable method is essentially a cable replacement technique, where we can achieve cable connection functionality, without actual RF cable connections. Therefore, antenna patterns are not inherently included, as in the conducted cable testing setup. We can embed the antenna patterns in the channel models in the performance testing once they are completely known in advance and static. However, this is a problem if UE antennas are adaptive, i.e. UE antennas can adapt to the RF propagation environments. The current discussion is limited to $2 \times 2$ MIMO terminals, and there are some logic extensions:

- We should investigate the applicability of the wireless cable method for high-order MIMO terminals.

- The wireless cable method is very attractive for largeobject equipped with a few non-adaptive antennas, e.g. automobile systems.
There are some challenges for applying the wireless cable method for $5 \mathrm{G}$ antenna systems:

- The cost setup is expensive, since the number of OTA antennas has to be no less than the number of DUT antennas.

- The calibration of transfer matrix between OTA antenna port and DUT antenna port, if possible at all, might be difficult and time-consuming.

- The wireless cable method is not well suited for adaptive antenna systems.

\section{ACKNOWLEDGMENT}

Dr. Wei Fan would like to acknowledge the financial assistance from Danish council for independent research (DFF611100525). This work has been partially supported by the Innovation Fund Denmark via the Virtusuo project.

\section{REFERENCES}

[1] Keysight Technologies, “Testing 5g: Evolution or revolution?" Tech. Rep., October 2016.

[2] Keysight Technologies, "Modelling what matters: Keeping a correct focus on 5g," Tech. Rep., October 2016.

[3] Microwave Vision Group (MVG), "Over-the-air testing of receive diversity and mimo capable terminals," Tech. Rep., July 2015.

[4] "Test Plan for 2x2 Downlink MIMO and Transmit Diversity Over-theAir Performance," CTIA Certification, Tech. Rep. Version 1.0, August 2015.

[5] "Verification of radiated multi-antenna reception performance of User Equipment (UE)," 3rd Generation Partnership Project, Tech. Rep. 3GPP TR 37.977 V14.0.0, June 2016.

[6] Keysight Technologies, "Mimo over the air (ota) handset performance and testing," Tech. Rep., July 2015.

[7] P. Kyösti, T. Jämsä, and J.-P. Nuutinen, "Channel modelling for multiprobe over-the-air MIMO testing," International Journal of Antennas and Propagation, vol. 2012, 2012.

[8] W. Fan, P. Kyösti, L. Hentilä, and G. F. Pedersen, "Mimo terminal performance evaluation with a novel wireless cable method," IEEE Transactions on Antennas and Propagation, vol. PP, no. 99, pp. 1-1, 2017.

[9] Y. Jing, H. Kong, and M. Rumney, "Mimo ota test for a mobile station performance evaluation," IEEE Instrumentation Measurement Magazine, vol. 19, no. 3, pp. 43-50, June 2016.

[10] W. Yu, Y. Qi, K. Liu, Y. Xu, and J. Fan, "Radiated Two-Stage Method for LTE MIMO User Equipment Performance Evaluation," IEEE Transactions on Electromagnetic Compatibility, vol. 56, no. 6, pp. 16911696, Dec 2014.

[11] C. Schirmer, M. Lorenz, W. A. T. Kotterman, R. Perthold, M. H Landmann, and G. D. Galdo, "MIMO over-the-air testing for electrically large objects in non-anechoic environments," in 2016 10th European Conference on Antennas and Propagation (EuCAP), April 2016, pp. $1-6$.

[12] Y. Jing, H. Kong, and M. Rumney, "Radiated two-stage mimo ota test method progress for antenna performance evaluation," in 2016 Asia-Pacific International Symposium on Electromagnetic Compatibility (APEMC), vol. 01, May 2016, pp. 729-731.

[13] M. Rumney, H. Kong, and Y. Jing, "Practical active antenna evaluation using the two-stage mimo ota measurement method," in The 8th European Conference on Antennas and Propagation (EuCAP 2014), April 2014, pp. 3500-3503.

[14] M. Rumney, H. Kong, Y. Jing, Z. Zhang, and P. Shen, "Recent advances in the radiated two-stage MIMO OTA test method and its value for antenna design optimization," in 2016 10th European Conference on Antennas and Propagation (EuCAP), April 2016, pp. 1-5.

[15] M. Rumney, H. Kong, Y. Jing, and X. Zhao, "Advances in antenna pattern-based MIMO OTA test methods," in 2015 9th European Conference on Antennas and Propagation (EuCAP), May 2015, pp. 1-5. 